\title{
Dynamic Study of a Capacitive MEMS Switch with Double Clamped-Clamped Microbeams
}

\author{
Hatem Samaali, ${ }^{1}$ Fehmi Najar, ${ }^{1}$ and Slim Choura ${ }^{2}$ \\ ${ }^{1}$ Applied Mechanics and Systems Research Laboratory, Tunisia Polytechnic School, University of Carthage, \\ BP 743, 2078 La Marsa, Tunisia \\ ${ }^{2}$ Research Group on Intelligent Machines, National Engineering School of Sfax, University of Sfax, BP 1173, 3038 Sfax, Tunisia
}

Correspondence should be addressed to Fehmi Najar; fehmi.najar@gmail.com

Received 5 July 2012; Accepted 2 November 2012; Published 27 March 2014

Academic Editor: Sami El-Borgi

Copyright (C) 2014 Hatem Samaali et al. This is an open access article distributed under the Creative Commons Attribution License, which permits unrestricted use, distribution, and reproduction in any medium, provided the original work is properly cited.

\begin{abstract}
We study a capacitive MEMS switch composed of two clamped-clamped exible microbeams. We first develop a mathematical model for the MEMS switch where the upper microbeam represents the ground transmission line and the lower one represents the central transmission line. An electrostatic force is applied between the two microbeams to yield the switch to its ON and OFF states. We derive the equations of motion of the system and associated boundary conditions and solve the static and dynamic problems using the differential quadratic method. We show that using only nine grid points gives relatively accurate results when compared to those obtained using FEM. We also examine the transient behavior of the microswitch and obtain results indicating that subsequent reduction in actuation voltage, switching time, and power consumption are expected along with relatively good RF performances. ANSYS HFSS simulator is used in this paper to extract the RF characteristics of the microswitch. HFSS simulation results show that the insertion loss is as low as $-0.31 \mathrm{~dB}$ and that the return loss is better than $-12.41 \mathrm{~dB}$ at $10 \mathrm{GHz}$ in the ON state. At the OFF state, the isolation is lower than $-23 \mathrm{~dB}$ in the range of 10 to $50 \mathrm{GHz}$.
\end{abstract}

\section{Introduction}

In telecommunication, MEMS devices offer a multitude of components to replace the classical semiconductor circuits elements. Microswitches and microresonators are used in a series of applications extending from the mobile phone and wireless networks to fiber-optic communication and multiplexed networks [1-3]. The major tasks of these devices are switching, filtering, and tuning. The equivalent circuit elements to these devices (PIN diode and Field-Effect Transistors FET) are generally characterized by high power consumption, low reliability, and high-manufacturing costs. In addition, they present unsatisfactory performance for high signal frequencies [4]; they give a high insertion loss and inadequate isolation at $\mathrm{ON}$ and $\mathrm{OFF}$ switching state.

Radio frequency MEMS (RF-MEMS) components have been recently widely developed and used in several applications. In particular, RF-MEMS microswitches are used in telecommunication applications to replace the traditional microelectronic switches (diodes and transistors). These microswitches present an improved insertion loss and good isolation during the "ON/OFF" switching states [4]. However, they are limited by the high actuation voltage (up to 30 volts) and slow switching time (nearly 300 microseconds). As a result, several researchers want to ameliorate the switching time, minimize the actuation voltage, and integrate RFMEMS switches with IC [5]. To satisfy this integration, RF MEMS switches must satisfy the following conditions: (a) have very small size, (b) have low actuation voltage, and (c) present low power consumption.

Several mechanisms of actuation have been used to actuate RF-MEMS switches such as electromagnetic [6], electrostatic [7], thermal [8], and piezoelectric [9]. Due to its simplicity of integration, electrostatic actuation represents the most used actuation technique at the present time [10]. This is also due to its low power consumption, as well as small electrode size, in addition it is easy to be integrated with 
IC circuits and it provides short switching time compared with the other mechanisms. However, it requires relatively high DC voltage, and thus, requires an additional CMOS integrated upconverter to raise the typical $5 \mathrm{~V}$ control voltage to the required level.

Electrostatically actuated structures present several nonlinearities and can become unstable. These nonlinearities are mainly due to the nature of the electrostatic force which is inversely proportional to the square of the distance between the two electrodes. A sudden collapse of the moving part can be observed at critical voltage (pull-in) of the microswitch achieving its ON or OFF state. In the literature the pullin instability is classified into static and dynamic pull-in. Static pull-in occurs when the DC voltage exceeds a threshold value with maximum displacements varying from $33 \%$ to $41 \%$ of the original electrode gap distance. On the other hand, dynamic pull-in takes place when the system is excited using a combination of AC and DC voltages. In this case, the dynamic pull-in instability occurs before static pullin [11, 12]. Nayfeh et al. [13] showed that dynamic pullin occurs at voltages as low as $25 \%$ of the static pull-in voltage around the resonant frequency. Recently Khater et al. [14] validated experimentally this approach; they developed dynamic actuation methods based on frequency sweep to shunt a capacitive microswitch. The proposed technique lead to $60 \%$ reduction of the actuation voltage of the microswitch.

The decrease of actuation voltage of electrostatic RFMEMS switches can be accomplished by (i) using different mechanical properties which reduce the microbeam rigidity, (ii) increasing the electrostatic surface, and/or (iii) decreasing the electrostatic gap. These variations degrade the principal parameters of the RF-MEMS switches, such as isolation. Abbaspour-Sani and Afrang proposed the decrease of the equivalent rigidity of the microswitch whose structure is composed of two displaceable microplates [15]; this preserves the microswitch parameters while increasing its lifetime. Similarly, Chaffey and Austin [16] decreased the equivalent rigidity of the microsystem and concluded that the use of a double cantilever microbeam structure, compared to a single microbeam structure, reduces significantly the pullin voltage. Recently Samaali et al. [17] demonstrated that when we use a double cantilever microbeam to design an RF microswitch, a reduction of the pull-in voltage, the switching time and the power consumption are observed.

The present paper examines the static and dynamic behaviors of an electrostatically actuated capacitive contact RF microswitch. The proposed design consists of a pair of bridge microbeams with clamped ends. An electrostatic force is applied between the two microbeams (electrodes) causing their deflections and the collapse of the upper microbeam onto the lower one when the pull-in voltage is reached. We investigate the static and transient responses of the RF microswitch as we vary the applied DC voltage and study its $\mathrm{ON}-\mathrm{OFF}$ cycle. The power requirement of the actuation cycle is also studied and compared with the classical single beam design.

\section{Model Description and Reduced-Order Model}

2.1. Problem Formulation. The schematic view of the capacitive microswitch is given in Figure 1. It consists of double suspended bridges that represent the ground and the central transmission lines. Both upper and lower beams are clamped at their ends to the substrate. A dielectric layer is used to separate the electrodes and prevent electrical short circuit. The microbeams are modeled using Euler-Bernoulli beam theory using the following nondimensional coupled-integralpartial-differential equations and their associated boundary conditions [18]:

$$
\begin{gathered}
\ddot{w}_{i}+c_{i} \dot{w}_{i}+w_{i}^{\prime \prime \prime \prime} \\
=\left[F_{i}+\alpha_{1} \int_{0}^{1}\left(w_{i}\left(x_{i}, t\right)\right)^{2} d x_{i}\right] w_{i}^{\prime \prime} \\
+\alpha_{2} \frac{V_{\mathrm{DC}}^{2}}{\left(1-d_{w}\right)^{2}}, \quad i=1,2 \\
w_{i}(0, t)=0, \quad w_{i}(1, t)=0, \\
w_{i}^{\prime}(0, t)=0, \quad w_{i}^{\prime}(1, t)=0,
\end{gathered}
$$

where $w_{i}\left(x_{i}, t\right)$ is the nondimensional deflection of each microbeam at the nondimensional time $t$ and at nondimensional locations $x_{i}$ and $d_{w}=w_{1}\left(x_{1}, t\right)+w_{2}\left(x_{2}, t\right)$. The dot denotes the derivative with respect to $t$ and the prime derivatives with respect to $x_{i} \cdot c_{i}$ represents the beam's nondimensional damping related to the quality factor by $Q=$ $\omega / c_{i}, \omega$ is the nondimensional natural frequency, $F_{i}$ is the nondimensional axial force generated by residual stresses, $V_{\mathrm{DC}}$ is the DC voltage, and $L$ is the length of both microbeams. The variables are nondimensionalized using the following form:

$$
\begin{array}{ll}
w_{i}=\frac{\widehat{w}_{i}}{d}, \quad x_{i}=\frac{\widehat{x}_{i}}{L}, \quad t=\frac{\widehat{t}}{\tau}, & \alpha_{1}=6\left(\frac{d}{h}\right)^{2}, \\
\alpha_{2}=6\left(\frac{\epsilon L^{4}}{E d^{3} h^{3}}\right), & F_{i}=\widehat{F}_{i} \frac{L^{2}}{E I}, \quad \tau=\sqrt{\frac{L^{4} \rho A}{E_{f} I}},
\end{array}
$$

where the hats denote the corresponding dimensional quantities. Here $\rho$ is the density, $E$ is the modulus of elasticity, $b, h, A=b h$, and $I=b h^{3} / 12$ are beam's width, thickness, cross-section area, and second moment of area, respectively. $d$ is the initial gap distance between both microbeams and $h_{d}$ is the dielectric layer thickness. The geometric and physical parameters of the microswitch are given in Table 1.

2.2. Discretization and Reduced-Order Model. DQM transforms a PDE into a set of ODEs describing the motion of a set of prespecified discrete points (a grid) corresponding to discrete values of the continuous space variable. This is accomplished by expressing, at each grid point, the derivative of the deflection function with respect to the space variable 
TABLE 1: Geometric and physical parameters of the beam.

\begin{tabular}{lccccccc}
\hline$L$ & $b$ & $h$ & $d$ & $\rho$ & $E$ & $\varepsilon$ & $h_{d}$ \\
\hline $510 \mu \mathrm{m}$ & $100 \mu \mathrm{m}$ & $1.5 \mu \mathrm{m}$ & $1.18 \mu \mathrm{m}$ & $2300 \mathrm{Kg} / \mathrm{m}^{3}$ & $166 \mathrm{GPa}$ & $8.85110^{-12} \mathrm{~F} / \mathrm{m}$ & $0.236 \mu \mathrm{m}$ \\
\hline
\end{tabular}

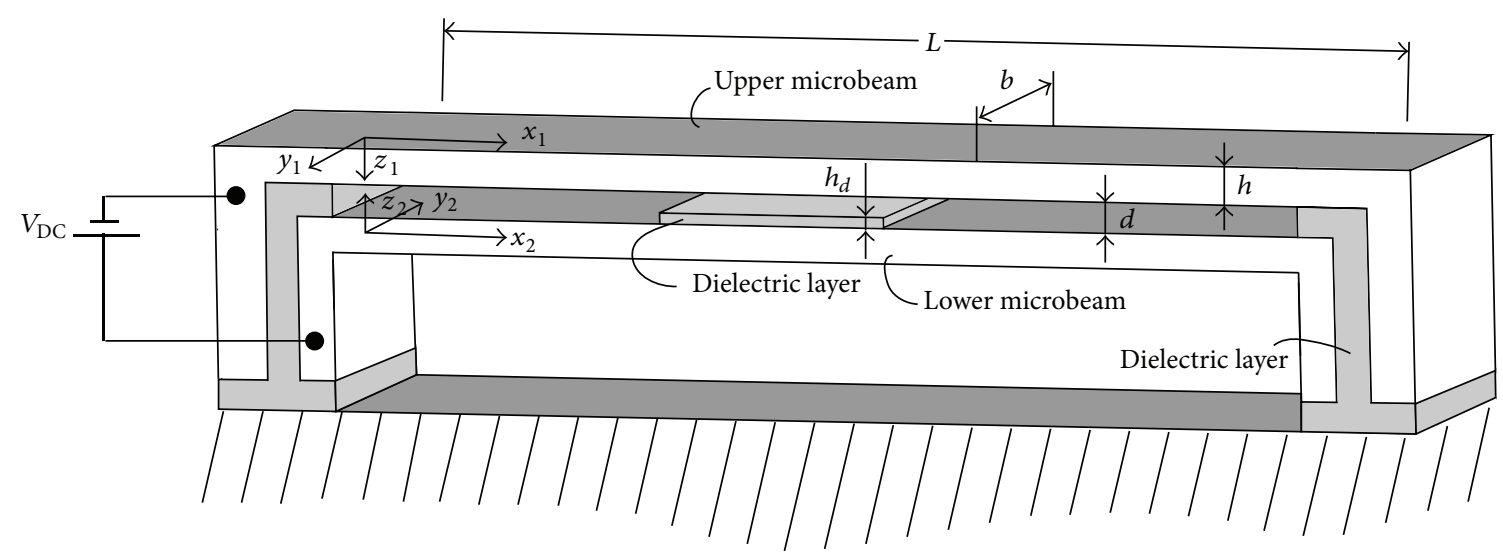

FIGURE 1: Capacitive contact RF-MEMS switch.

as a weighted linear sum of the values of this function at all $n$ grid points [19]. That is,

$$
\left[\frac{\partial f^{r}(x)}{\partial x^{r}}\right]_{x=x_{i}}=\sum_{j=1}^{n} A_{i j}^{(r)} f_{j}
$$

where $f_{j}=f\left(x_{j}\right)$, the $x_{j}$ are the Chebyshev-Gauss-Lobatto grid points defined by [19] $x_{i}=(1 / 2)[1-\cos ((i-1) /(n-$ $1)) \pi]$, and $A_{i j}^{(r)}$ is the weighting coefficient of the $r$ thorder derivative. These coefficients are determined using the Lagrange interpolation polynomials as test functions in (3). They are given by $[20,21]$

$$
\begin{gathered}
A_{i j}^{(1)}=\left(\frac{\prod_{v=1 ; v \neq i}^{n}\left(x_{i}-x_{v}\right)}{\left(x_{i}-x_{j}\right) \prod_{v=1 ; v \neq j}^{n}\left(x_{j}-x_{v}\right)}\right), \\
i, j=1,2, \ldots, n, \quad j \neq i, \\
A_{i j}^{(r)}=r\left(A_{i i}^{r-1} A_{i j}^{1}-\frac{A_{i j}^{(r-1)}}{\left(x_{i}-x_{j}\right)}\right), \\
i, j=1,2, \ldots, n, \quad j \neq i, \\
A_{i i}^{(r)}=-\sum_{v=1 ; v \neq i}^{n} A_{i v}^{(r)}, \quad i=1,2, \ldots, n,
\end{gathered}
$$

where $\left[A^{(r)}\right]$ is the matrix corresponding to the $r$ th-order derivative. These matrices are centrosymmetric when $r$ is even and skew-centrosymmetric when $r$ is odd.

According to the previously published works [18, 22], the DQM is a suitable method to produce reduced-order models (ROM) in the case of clamped-clamped microbeam with nonlinear electrostatic force. We use $n$ grid points to discretize the space and obtain a ROM given by the following $n-3$ coupled second-order ordinary differential equations
(ODEs) and associated boundary conditions, describing the motion of the microswitch:

$$
\begin{gathered}
\ddot{w}_{i k}+c_{i} \dot{w}_{i k}+\sum_{j=1}^{n} A_{j k}^{(4)} w_{i j}=\left[F_{i}-\alpha_{1} \sum_{j=1}^{n} \sum_{l=1}^{n} C_{j} w_{i j} A_{j k}^{(2)} w_{i l}\right] \\
\times \sum_{j=1}^{n} A_{j k}^{(2)} w_{i j}+\alpha_{2} \frac{V_{\mathrm{DC}}^{2}}{\left(1-d_{w}\right)^{2}}, \\
k=3, \ldots, \frac{n+1}{2}, \quad i=1,2, \\
w_{i 1}=0, \quad w_{i n}=0, \quad \sum_{j=1}^{n} A_{1 j}^{(1)} w_{i j}=0, \\
\sum_{j=1}^{n} A_{n j}^{(1)} w_{i j}=0,
\end{gathered}
$$

where $d_{w}=w_{1 k}+w_{2 k}, w_{i k}=w_{i}\left(x_{k}, t\right)$. The associated static algebraic nonlinear system is solved using the NewtonRaphson in Mathematica. The transient analysis is obtained using Long Time Integration (LTI) obtained by the RungeKutta method.

\section{Response of the Microswitch under DC Voltage}

3.1. Static Response under DC Voltage. Figure 2 shows the variation of the static deflections at the center of the microbeams as the applied DC voltage is increased. Here due to symmetry of the problem only the deflection of one microbeam will be shown. The static response is obtained using the DQM (9 grid points) and validated with finite element (FE) analysis of the static responses obtained using 


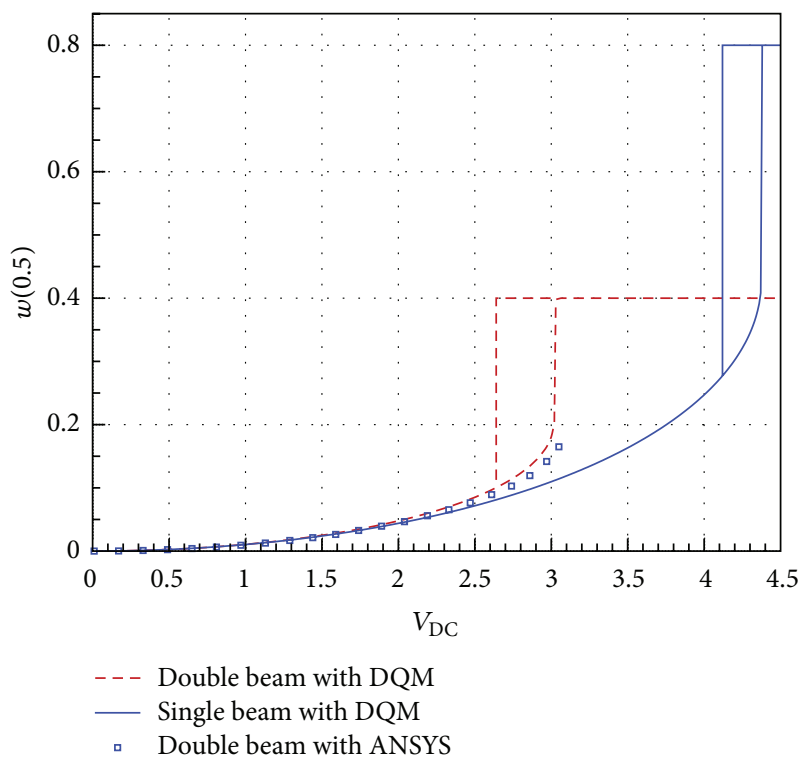

FIGURE 2: Microbeams deflection under an applied DC voltage and validation with ANSYS results.

the commercial software ANSYS. A comparison of the static response of the proposed system with a classical microswitch using one microbeam is shown in Figure 2. The computed static pull-in voltages associated with the single and double microbeams are $3.01 \mathrm{~V}$ and $4.38 \mathrm{~V}$, respectively. Consequently for the static analysis a $32 \%$ reduction of the actuation voltage is observed. However, the pull-out voltage, also shown in Figure 2, requires more voltage reduction than the single beam case. In fact, the required voltage reduction is $0.37 \mathrm{~V}$ for the double beam case, while it requires only $0.26 \mathrm{~V}$ reduction for the single case to pull-out. This difference is due to the overall stiffness reduction of the system.

3.2. Transient Response under DC Voltage. The transient analysis of the microswitch under different applied DC voltages are shown in Figure 3. Long time integration is used here with the Runge-Kutta discretization technique to calculate the transient response of one of the two microbeams to a step DC voltage. In Figure 3 we show variation of the transient response as we vary the applied DC voltage. As shown the solution diverges as the applied voltage reaches $2.75 \mathrm{~V}$ and the microbeam pulls-in. Here we observe a difference between the static pull-in value $(3.01 \mathrm{~V})$ and the transient pull-in value $(2.75 \mathrm{~V})$, this mismatch is essentially due to the inertial effects of the microbeam, not taking into account the static analysis.

\section{Pull-In Time and Power Requirement Using Transient Pull-In}

Switching time and power consumption (switching power) are important parameters in RF MEMS Switches, and they constitute the main limitation for microswitches [23]. When the applied voltage increases the electrostatic force becomes higher than the elastic restoring force of the microbeam and the upper beam touches the lower beam causing pull-in. The time taken by the switch to pull-in is called the switching time or the pull-in time. Here in the proposed design each microbeam travels only $50 \%$ of the gap distance to reach the OFF state of the microswitch. Therefore, the switching time is shorter when compared to the single beam design.

The mean value of the electrostatic power $P$ is defined by (6). This power corresponds to the mean value of the sum of instantaneous electrostatic power over the pull-in time $T_{s}$, given by the approximated parallel capacitances shown in Figure 4 . The chosen capacitances are related to the selected DQM grid points to discretize the equation of motion. Equation (6) is then solved numerically at each time instant for the instantaneous power then integrated over the pull-in time period. $C_{k}$ is the discretized capacitance for a given applied voltage.

$$
P=\sum_{i=2}^{n-1} P_{i}=P_{2}+\frac{1}{T_{s}} \sum_{k=3}^{n-2} \int_{0}^{T_{s}} C_{i} d V_{\mathrm{DC}}^{2} \dot{w}_{k} d \widehat{t}+P_{n-1},
$$

where

$$
\begin{gathered}
P_{2}=\frac{1}{2 T_{s}} \int_{0}^{T_{s}} \frac{\varepsilon b L}{\left(1-w_{2}\right)}\left(x_{3}+x_{2}\right) V_{\mathrm{DC}}^{2} \dot{w}_{2} d \widehat{t}, \\
P_{n-1}=\frac{1}{T_{s}} \int_{0}^{T_{s}} \frac{\varepsilon b L}{\left(1-w_{n-1}\right)}\left(x_{n}-\frac{x_{n-1}+x_{n-2}}{2}\right) V_{\mathrm{DC}}^{2} \dot{w}_{n-1} d \widehat{t}, \\
C_{i}=\frac{\varepsilon b L}{d\left(1-w_{i}\right)}\left(\frac{x_{i+1}-x_{i-1}}{2}\right) .
\end{gathered}
$$

In Figure 5 we study the influence of the applied DC voltage on the pull-in time. In this figure, we show that this latter is reduced significantly by increasing the applied voltage in both single and double beam configurations. It is clear that using DC voltage to actuate the microswitch offers significantly improved performances for the double beam design.

We examine also the electrostatic power requirement of both designs. We note, in Figure 6, that the double beam switch requires lower actuated power when compared to the single beam design. Also we remark in Figure 6 that the minimum electrostatic power is obtained at $4.22 \mathrm{~V}$ for the single beam design and $2.92 \mathrm{~V}$ for the double beam design which is higher than the transient pull-in voltage in both cases. In Figure 7 we show the variation of the electrostatic power versus the pull-in time, as observed for the minimum used power in the single and double beam designs; almost the same transient pull-in time is obtained. However, the power used in this case is lower for the double beam designs.

\section{Electromagnetic Model Analysis}

In this section, we focus on the shunt capacitive MEMS switch behavior described above. The full wave electromagnetic simulation of the switch is done using Ansys HFSS. Sparameters are extracted in the frequency range going from $0.1 \mathrm{GHz}$ to $50 \mathrm{GHz}$ for different positions of the microswitch. 


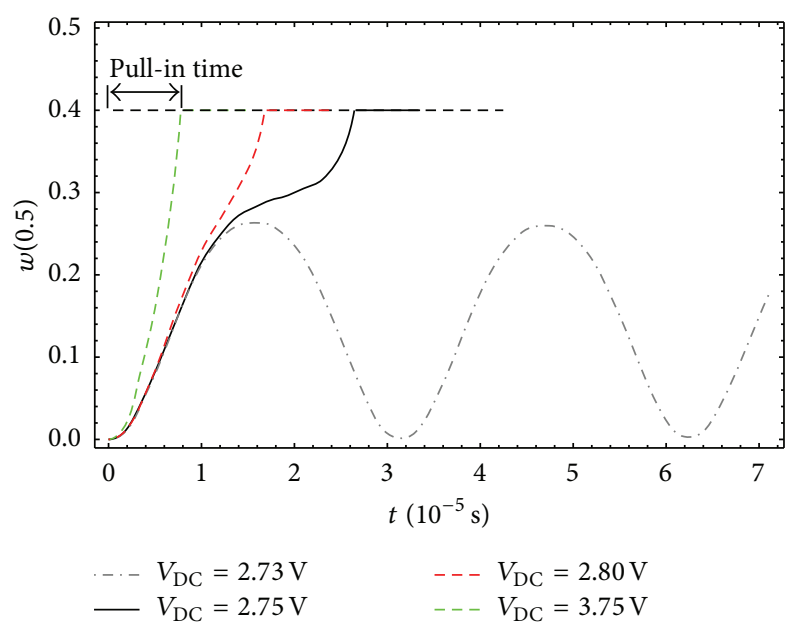

FIgURE 3: Transient response of the microswitch for different applied DC voltages.

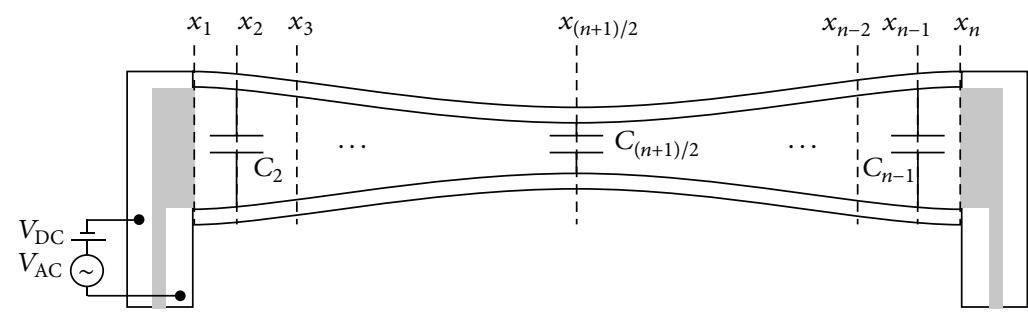

FIGURE 4: The equivalent parallel capacitances model of the capacitive microswitch.

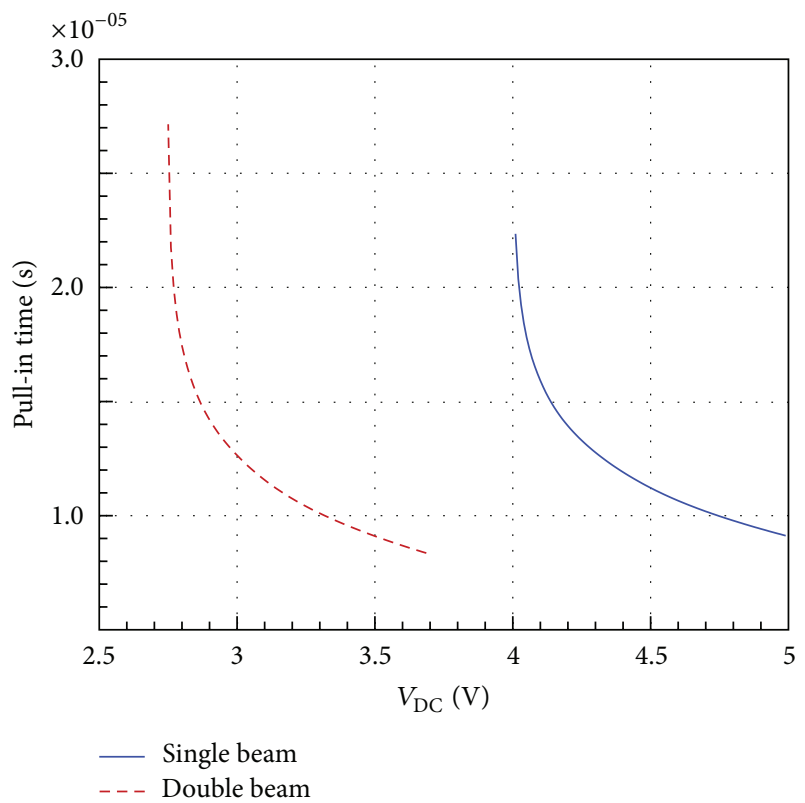

FIGURE 5: Influence of DC voltage on the pull-in time for simple and double beam microswitch designs.

In the simulation an air box size of $710 \times 1000 \times 900 \mu \mathrm{m}$ is used and boundary radiation conditions are imposed on the four sides of the box, two waves ports are placed in the

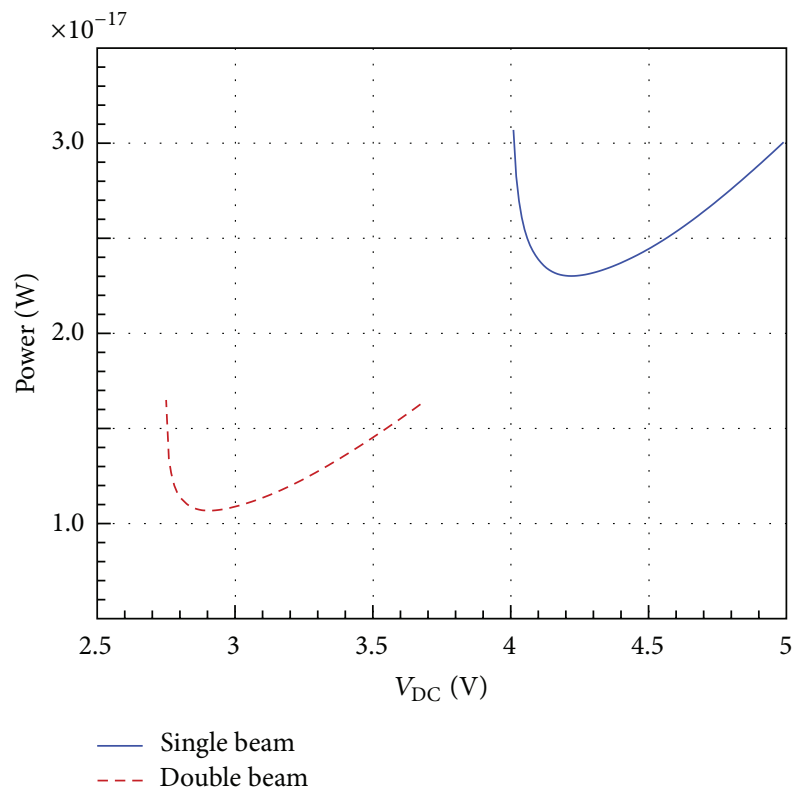

Figure 6: Influence of DC voltage on the electrostatic energy for simple and double beam microswitch designs.

two other sides of the box. The wave ports are the boundary condition that permits energy to flow into and from the microswitch's structure. 


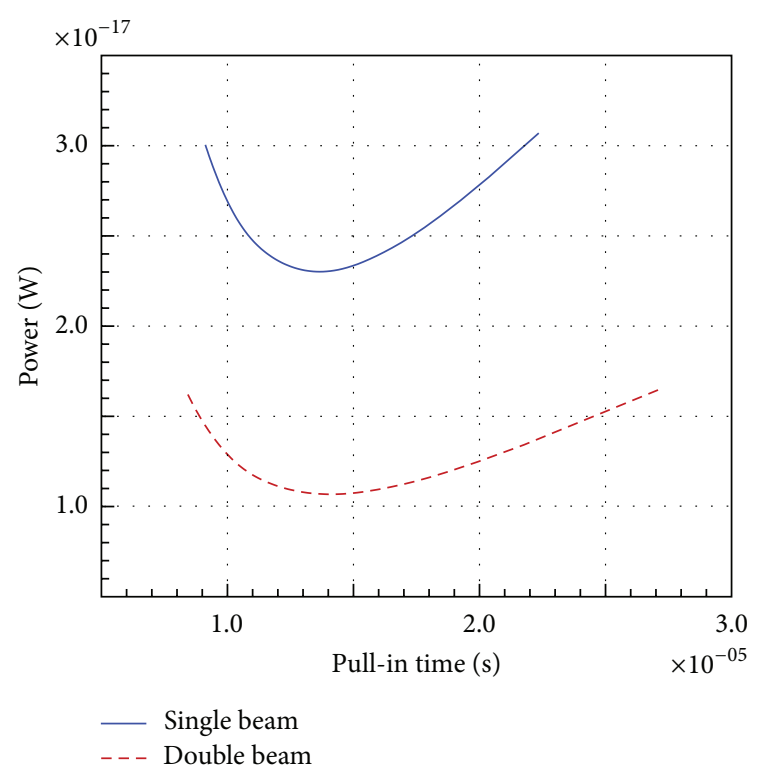

FIGURE 7: Variation of the electrostatic energy versus the pullin time by varying the DC voltage for simple and double beam microswitch designs.

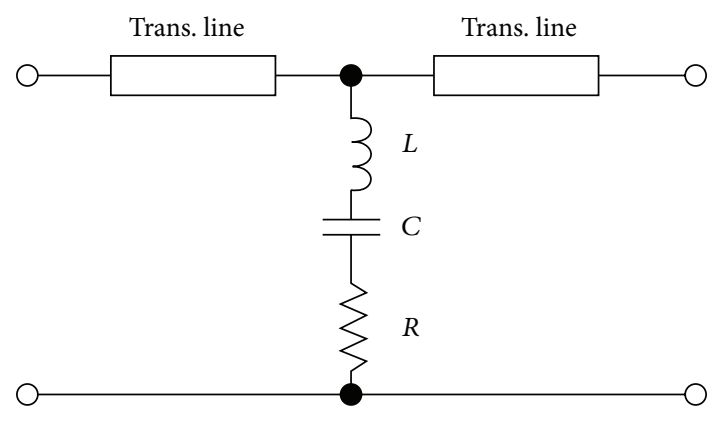

FIgURE 8: The equivalent circuit of the microswitch.

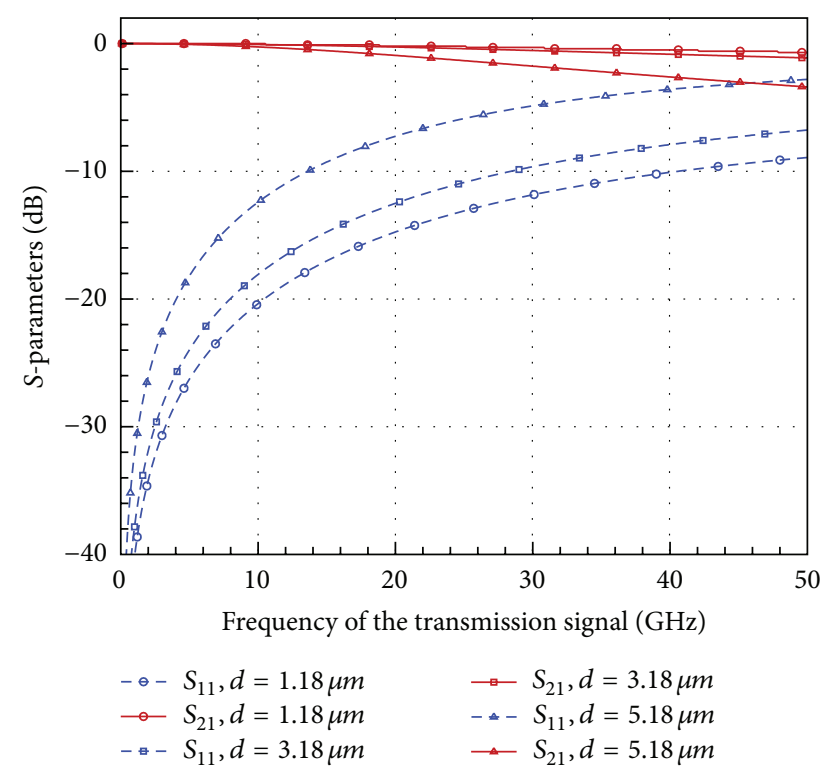

FIGURE 9: HFSS simulations results of the switch in ON state.

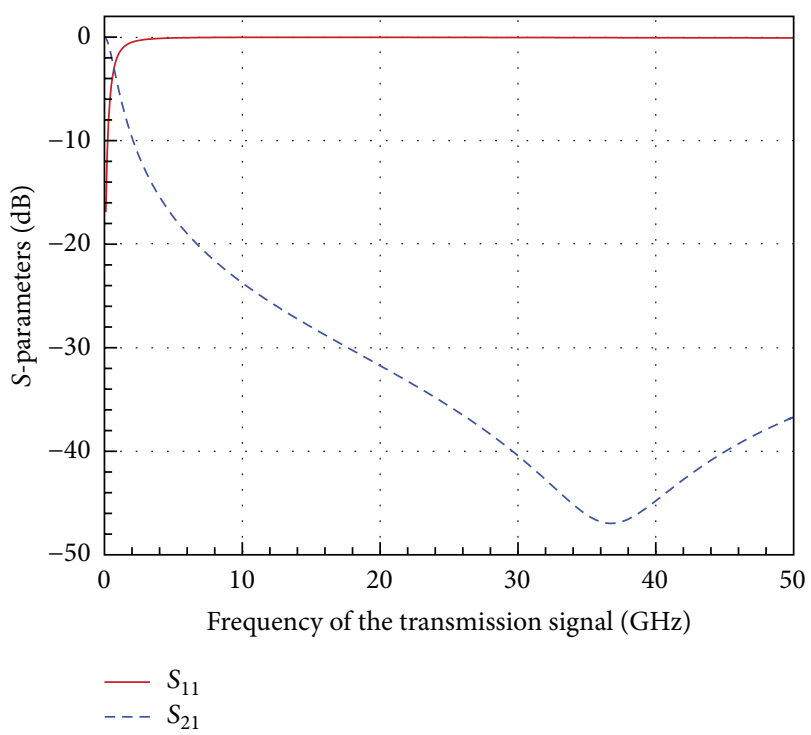

FIGURE 10: HFSS Simulation results of the switch in OFF state.

The used substrate has a relative dielectric constant correspondent to Silicon. The thickness of the substrate is $100 \mu \mathrm{m}$. The CPW conductors (ground and center transmission lines) are supposed to be perfect conductors. The central transmission line conductor of the CPW is assumed to be coated with silicon nitrate $\left(\mathrm{Si}_{3} \mathrm{~N}_{4}\right)$ having relative dielectric constant of 7 and thickness of $0.236 \mu \mathrm{m}$. The microswitch is modeled by two short sections of transmission lines with characteristic impedance $Z_{0}$, and a lumped resistance-inductancecapacitance (RLC) model of the bridge with capacitance having an upstate or downstate value. The equivalent circuit of the switch is shown in Figure 8. In the upstate, the switch behaves as a capacitor when the frequency is below the inductance-capacitance (LC) series resonant frequency and as an inductor when the frequency is above the LC series resonant frequency, and at resonance, it reduces to pure resistance [24]. The transmission coefficients $\left(S_{12}\right.$ and $\left.S_{21}\right)$ are commonly called gain or attenuation, and the reflection coefficients $\left(S_{11}\right.$ and $\left.S_{22}\right)$ are directly related to impedance [25]. In the ON state the value of the coupling capacitance is very small due to the large separation between upper and lower beams. The S-parameters $\left(S_{21}\right.$ and $\left.S_{11}\right)$ were extracted in the range 0.1 to $50 \mathrm{GHz}$.

In Figure 9 the insertion loss $\left(S_{21}\right)$ of the microswitch in the $\mathrm{ON}$ state is varied from $-0.31 \mathrm{~dB}$ to $-0.1 \mathrm{~dB}$ at $10 \mathrm{GHz}$ with gap distance $d$ varying from 1.18 to $5.18 \mu \mathrm{m}$. The return loss $\left(S_{11}\right)$ varies from -12.41 to $-20.36 \mathrm{~dB}$ at $10 \mathrm{GHz}$ for the same range of the gap $d$. We conclude that as we increase the distance that separates upper and lower beams, we minimize the coupling capacitance and we increase and decrease, respectively, the return loss and the insertion loss. With a sufficient applied voltage, the ground transmission line and central transmission line of the microswitch deflect and create a large coupling capacitance; the microswitch is in the OFF state. The isolation $\left(S_{21}\right)$ is lower than $-23 \mathrm{~dB}$ in the range of 10 to $50 \mathrm{GHz}$, as shown in Figure 10. 


\section{Conclusion}

We propose a design of a capacitive RF microswitch with two flexible microbeams. The new design allows a $32 \%$ reduction in the actuation voltage compared to a classical single beam design. A mathematical model was developed to analyze the static and transient behavior under different applied DC voltages. We observe that transient pull-in occurs before static pull-in at voltage as low as $8.6 \%$. Then we studied and compared the switching times and switching power for both single and double beam designs under different DC actuations. We showed that the new double beam design gives significant improvement in switching time and power requirement. Finally, the Ansys HFSS simulator is used to extract the RF characteristics of the switch. Simulations results showed that an insertion loss of $-0.31 \mathrm{~dB}$ and a return loss of $-12.41 \mathrm{~dB}$ at $10 \mathrm{GHz}$ for gap distance of $1.18 \mu \mathrm{m}$ have been achieved in the ON state. These results can be ameliorated by increasing the gap distance. At the OFF state isolation lower than $-23 \mathrm{~dB}$ in the range of 10 to $50 \mathrm{GHz}$ is extracted by the Ansys HFSS simulator.

\section{Conflict of Interests}

The authors declare that there is no conflict of interests regarding the publication of this paper.

\section{References}

[1] J. J. Yao, "RF MEMS from a device perspective," Journal of Micromechanics and Microengineering, vol. 10, no. 4, pp. R9R38, 2000.

[2] S. Lucyszyn, "Review of radio frequency microelectromechanical systems technology," IEE Proceedings: Science, Measurement and Technology, vol. 151, no. 2, pp. 93-103, 2004.

[3] J. A. Walker, "Future of MEMS in telecommunications networks," Journal of Micromechanics and Microengineering, vol. 10, no. 3, pp. R1-R7, 2000.

[4] G. M. Rebeiz, RF MEMS: Theory, Design, and Technology, Wiley-Interscience, Hoboken, NJ, USA, 2003.

[5] J. Y. Park, G. H. Kim, K. W. Chung, and J. U. Bu, "Monolithically integrated micromachined RF MEMS capacitive switches," Sensors and Actuators A: Physical, vol. 89, no. 1-2, pp. 88-94, 2001.

[6] J. A. Wrigth and Y. C. Tai, "Micro-miniature electromagnetic switches fabricated using MEMS technology," in Proceeding of the 46th Annual International Relay Confrence (NARM '98), pp. 13-1-13-4, Oak Brook, Ill, USA, 1998.

[7] P. M. Zavracky, S. Majumder, and N. E. McGruer, "Micromechanical switches fabricated using nickel surface micromachining," Journal of Microelectromechanical Systems, vol. 6, no. 1, pp. 3-9, 1997.

[8] D. Girbau, A. Lazaro, and L. Pradell, "RF-MEMS switches based on the buckle-beam thermal actuator," in Proceedings of the $3 \mathrm{rd}$ European Microwave Conference, vol. 2, pp. 651-654, 2003.

[9] S. J. Gross, Q. Q. Zhang, S. Trolier-McKinstry, S. Tadigadapa, and T. N. Jackson, "RF-MEMS piezoelectric switch," in Proceedings of the Device Research Conference, pp. 99-100, 2003.

[10] H. Samaali, F. Najar, S. Choura, A. H. Nayfeh, and M. Masmoudi, "Novel design of MEMS ohmic RF switch with low voltage actuation," in Proceedings of IEEE 3rd the International Conference on Signals, Circuits and Systems (SCS '09), Jerba, Tunisia, November 2009.

[11] R. K. Gupta, E. S. Hung, Y. J. Yang, G. K. Ananthasuresh, and S. D. Sentura, "Pullin dynamics of electrostatically actuated beams," in Proceedings of the Technical DigestSolid State Sensor and Actuator Workshop, pp. 1-2, 1996.

[12] G. N. Nielson and G. Barbastathis, "Dynamic pull-in of parallelplate and torsional electrostatic MEMS actuators," Journal of Microelectromechanical Systems, vol. 15, no. 4, pp. 811-821, 2006.

[13] A. H. Nayfeh, M. I. Younis, and E. M. Abdel-Rahman, "Dynamic pull-in phenomenon in MEMS resonators," Nonlinear Dynamics, vol. 48, no. 1-2, pp. 153-163, 2007.

[14] M. E. Khater, K. Vummidi, E. M. Abdel-Rahman, A. H. Nayfeh, and S. Raman, "Dynamic actuation methods for capacitive MEMS shunt switches," Journal of Micromechanics and Microengineering, vol. 21, no. 3, Article ID 035009, 2011.

[15] E. Abbaspour-Sani and S. Afrang, "A low voltage MEMS structure for RF capacitive switches," Progress in Electromagnetics Research, vol. 65, pp. 157-167, 2006.

[16] J. Chaffey and M. Austin, "Analytical modelling of the electromechanical coupling of cantilever beams," in Smart Structures, Devices, and Systems, vol. 4935 of Proceedings of SPIE, pp. 86-93, 2002.

[17] H. Samaali, F. Najar, S. Choura, A. H. Nayfeh, and M. Masmoudi, "A double microbeam MEMS ohmic switch for RFapplications with low actuation voltage," Nonlinear Dynamics, vol. 63, no. 4, pp. 719-734, 2011.

[18] F. Najar, A. H. Nayfeh, E. M. Abdel-Rahman, S. Choura, and S. El-Borgi, "Nonlinear analysis of MEMS electrostatic microactuators: primary and secondary resonances of the first mode," Journal of Vibration and Control, vol. 16, no. 9, pp. 13211349, 2010.

[19] C. W. Bert and M. Malik, "Semianalytical differential quadrature solution for free vibration analysis of rectangular plates," Journal of the American Institute of Aeronautics and Astronautics, vol. 34, no. 3, pp. 601-606, 1996.

[20] C. W. Bert and M. Malik, "Free vibration analysis of tapered rectangular plates by differential quadrature method: a semianalytical approach," Journal of Sound and Vibration, vol. 190, no. 1, pp. 41-63, 1996.

[21] S. Tomasiello, "Differential quadrature method: application to initial-boundary-value problems," Journal of Sound and Vibration, vol. 218, no. 4, pp. 573-585, 1998.

[22] F. Najar, S. Choura, S. El-Borgi, E. M. Abdel-Rahman, and A. H. Nayfeh, "Modeling and design of variable-geometry electrostatic microactuators," Journal of Micromechanics and Microengineering, vol. 15, no. 3, pp. 419-429, 2005.

[23] S. K. De and N. R. Aluru, "Full-Lagrangian schemes for dynamic analysis of electrostatic MEMS," Journal of Microelectromechanical Systems, vol. 13, no. 5, pp. 737-758, 2004.

[24] A. B. Yu, A. Q. Liu, Q. X. Zhang, A. Alphones, L. Zhu, and A. P. Shacklock, "Improvement of isolation for MEMS capacitive switch via membrane planarization," Sensors and Actuators A: Physical, vol. 119, no. 1, pp. 206-213, 2005.

[25] C.-L. Dai, H.-J. Peng, M.-C. Liu, C.-C. Wu, and L.-J. Yang, "Design and fabrication of RF MEMS switch by the CMOS process," Tamkang Journal of Science and Engineering, vol. 8, no. 3, pp. 197-202, 2005. 

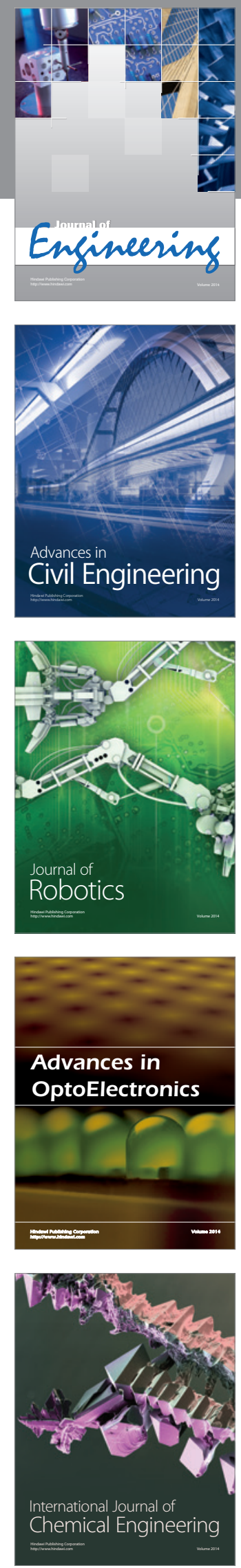

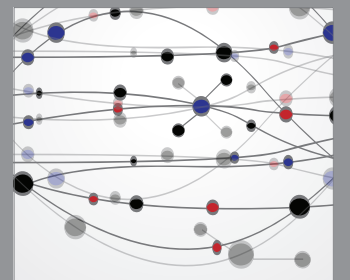

The Scientific World Journal
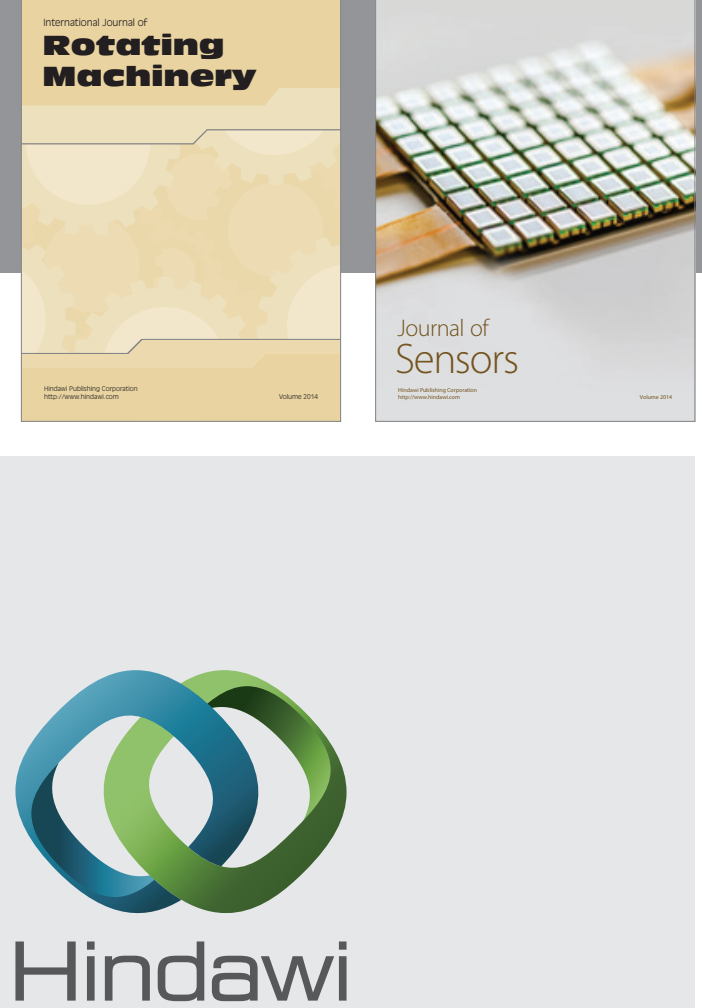

Submit your manuscripts at http://www.hindawi.com
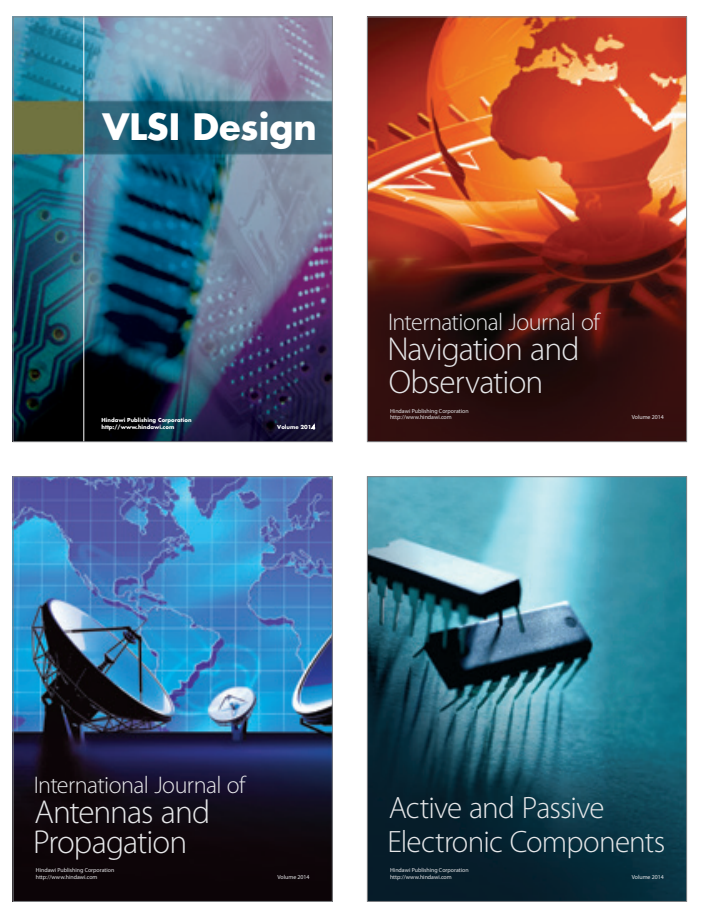
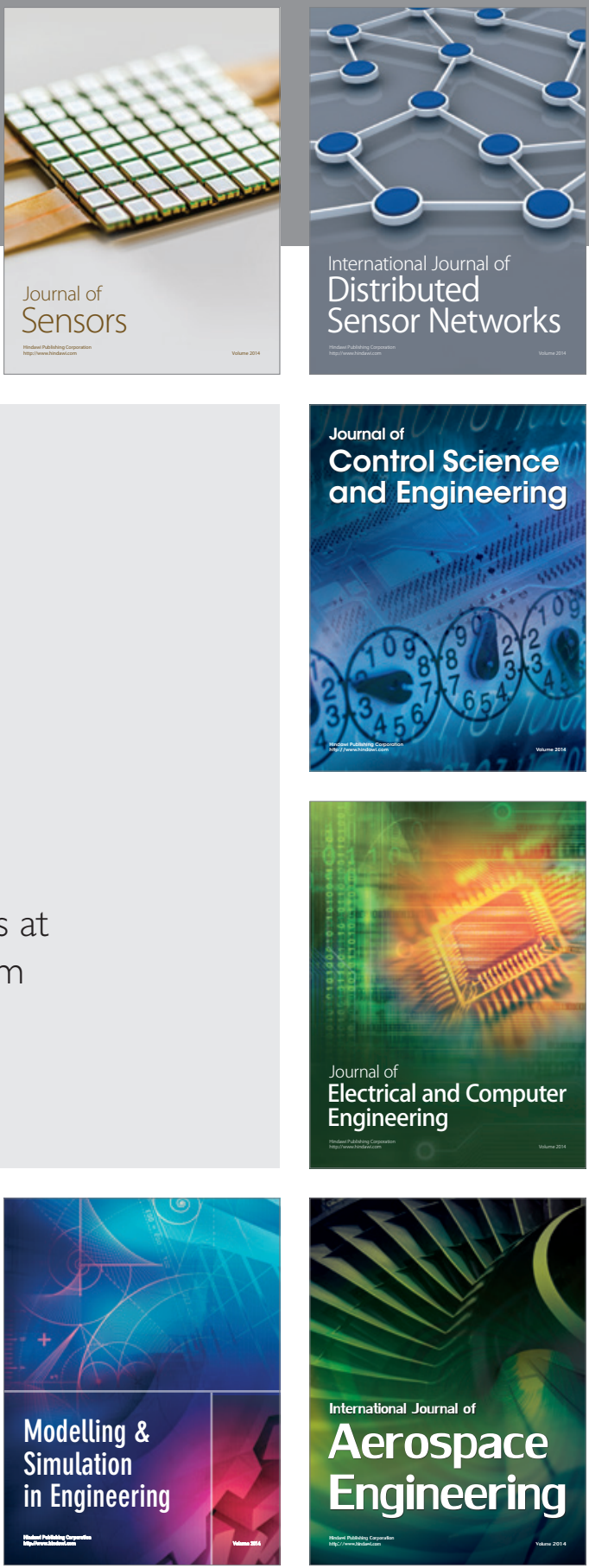

Journal of

Control Science

and Engineering
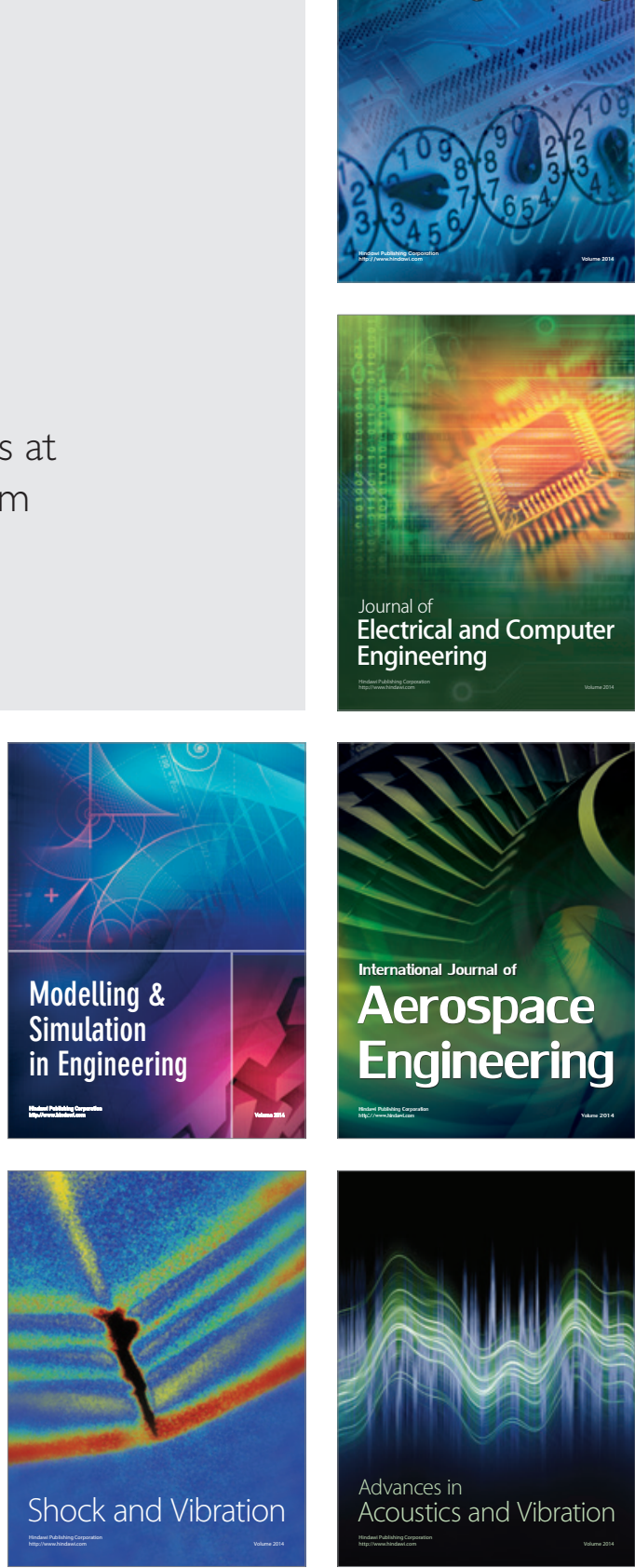